Open Access

\title{
Combined systemic and ocular chemotherapy for anterior segment metastasis of systemic mantle cell lymphoma
}

Aniruddha Agarwal' ${ }^{1}$ Mohammad Ali Sadiq' ${ }^{1}$ William R. Rhoades ${ }^{1}$, Loren S. Jack', Mostafa Hanout', Philip J. Bierman², William W. West ${ }^{3}$ and Quan Dong Nguyen ${ }^{1 *}$

\begin{abstract}
Background: Mantle cell lymphoma (MCL) is an aggressive subtype of non-Hodgkin's lymphoma that rarely metastasizes to the iris and the anterior segment. Blastic/pleomorphic morphology is thought to have an adverse effect on prognosis in MCL. MCL is resistant to conventional chemotherapeutic regimens with a tendency for multiple relapses. Management of anterior segment metastasis of systemic MCL has not been described in literature.

Findings: A 58-year-old male presented with an aggressive, relapsing, metastatic, systemic blastic variant of $\mathrm{MCL}$ with ocular involvement. At the time of initial presentation, large tumor cells were visible in the anterior chamber (AC) along with hypopyon and fibrin. The AC cells stained positively for CD20. The iris was thickened and coated with lymphoma cells. Iris neovascularization was present. Given extensive systemic and ocular involvement, the patient was given combination chemotherapy with systemic ibrutinib and intravitreal injections of methotrexate and rituximab. The disease response was monitored using multimodal imaging, including anterior segment optical coherence tomography and ultrasound biomicroscopy. Following combination of systemic and intraocular chemotherapy, there was a marked decrease in the ocular tumor load and the systemic disease.

Conclusions: Combination therapy with intravitreal injections of chemotherapeutic agents targeting monoclonal B-cell population and novel systemic agents may help to achieve remission in anterior segment metastasis of aggressive subtypes of NHL such as blastic variant of MCL. Multimodal imaging may assist in the management of these cases.
\end{abstract}

Keywords: Mantle cell lymphoma; Iris; Ibrutinib; Rituximab; Methotrexate; Metastasis; Uveitis; Ultrasound biomicroscopy

\section{Findings}

\section{Introduction}

Mantle cell lymphoma (MCL) is a rare subtype of non-Hodgkin's lymphoma (NHL) accounting for 6$7 \%$ of all NHL cases. Blastic/pleomorphic morphology is thought to have an adverse effect on prognosis in MCL. MCL is an aggressive form of NHL with poor response to conventional therapy and risk of multiple relapses [1, 2]. Systemic MCL can affect various organs such as lymph nodes, bone marrow, spleen, and bowel

\footnotetext{
* Correspondence: quan.nguyen@unmc.edu

'Stanley M. Truhlsen Eye Institute, University of Nebraska Medical Center, 985540 Nebraska Medical Center, Omaha 68198-5540, NE, USA Full list of author information is available at the end of the article
}

resulting in a poor median survival of 3-6 years after diagnosis [3]. However, systemic metastases of NHL to the eye are rare with most cases confined to the orbit and adnexa [4]. Intraocular involvement is even rarer, and it usually affects the choroid [5]. Thus, reports of iris and anterior segment involvement due to metastasis of systemic MCL have been limited to a few case reports [6-8].

While radiation therapy has been used for the management of orbit and adnexal involvement [9], intravitreal injections of chemotherapeutic agents such as methotrexate (MTX) and rituximab (anti-CD20) have been used for patients with vitreoretinal NHL $[10,11]$. The treatment for iris metastasis has not been well described

\section{Springer}

(c) 2015 Agarwal et al. Open Access This article is distributed under the terms of the Creative Commons Attribution 4.0 International License (http://creativecommons.org/licenses/by/4.0/), which permits unrestricted use, distribution, and reproduction in any medium, provided you give appropriate credit to the original author(s) and the source, provide a link to the Creative Commons license, and indicate if changes were made. 
in literature. Ibrutinib is a novel Bruton's tyrosine kinase (BTK) inhibitor recently introduced for the treatment of relapsed systemic MCL [12]. In this index case report, multimodal imaging analysis of a patient with anterior segment metastasis of relapsed systemic MCL is described. Treatment outcome using combination chemotherapy with systemic ibrutinib and intravitreal injections of MTX and rituximab is also discussed.

\section{Case report}

A 58-year-old Caucasian man was diagnosed with systemic MCL, blastic variant, in August 2010. He received six cycles of rituximab hyper-CVAD chemotherapy consisting of alternating combinations of drugs (course A and course B). Course A included cyclophosphamide, vincristine, doxorubicin, and dexamethasone; course B consisted of MTX and cytarabine [13]. Subsequently, the patient received autologous stem cell transplantation (ASCT) with BEAM chemotherapy [bis-chloroethylnitrosourea (carmustine), etoposide, cytarabine, and melphalan] in January 2012 [14]. Following ASCT, the disease was in remission.

A routine restaging positron emission tomography (PET) scan in January 2013 demonstrated relapse of the disease with new, enlarged mediastinal lymph nodes. Biopsy of a gluteal mass revealed metastatic MCL in March 2013. The patient received six cycles of bendamustine plus rituximab salvage [15]. A repeat PET scan in January 2014 did not show any disease activity.

In April 2014, the patient complained of redness, pain, and milky white deposits in the right eye (OD). He was diagnosed with anterior uveitis and elevated intraocular pressure (IOP) and was started on topical prednisone acetate $1 \%$ (four times a day) along with pilocarpine $2 \%$ three times a day. He underwent trabeculectomy and anterior chamber (AC) paracentesis in August 2014. The paracentesis sample was positive for CD20+ lymphoma cells. PET scan demonstrated several areas of nodal and extranodal disease (scalp mass and mediastinal nodes). Cerebrospinal fluid (CSF) and bone marrow biopsy, however, did not reveal lymphoma cells.

The patient presented to the Stanley M. Truhlsen Eye Institute at the University of Nebraska Medical Center in September 2014 with blurred vision, redness, and pain in OD. Best-corrected visual acuity (BCVA) was counting fingers at $3 \mathrm{~m}$ in OD and 20/20 in the left eye (OS). A hypopyon of large tumor cells filled more than half of the $\mathrm{AC}$; large keratic precipitates were seen on the inferior cornea. The iris was thickened and coated with lymphoma cells. There was extensive neovascularization of the iris (NVI). The angles were obliterated and the AC was shallow (Fig. 1a). Anterior segment optical coherence tomography (AS-OCT) (Heidelberg Spectralis ${ }^{\circ}$, Heidelberg, Germany) performed using the cornea protocol revealed large cells floating in the AC (Fig. 2a, b). There was marked thickening of the iris of OD compared to OS. Evaluation of the posterior segment with ultrasound B-scan demonstrated a clear vitreous cavity and absence of any retinochoroidal lesions. MRI revealed no orbital, optic nerve, brain parenchymal, or meningeal enhancement.

Due to the extensive ocular involvement and the malignant morphology of the CD20-positive cells, combination chemotherapy with intravitreal injections of MTX (400 $\mu \mathrm{g} / 0.1 \mathrm{ml})$ and rituximab $(1 \mathrm{mg} /$ $0.1 \mathrm{ml}$ ) were administered on two consecutive days. Intravitreal ranibizumab $(0.5 \mathrm{mg})$ was also administered for the NVI. The oncologist initiated systemic chemotherapy with ibrutinib $560 \mathrm{mg} /$ day. A repeat AC paracentesis was performed for cytological analysis, which revealed monoclonal B-cell lymphoma population positive for CD20, CD5, cyclin D1, and SOX11 but negative for CD10, CD3, and S100 (Fig. 3).
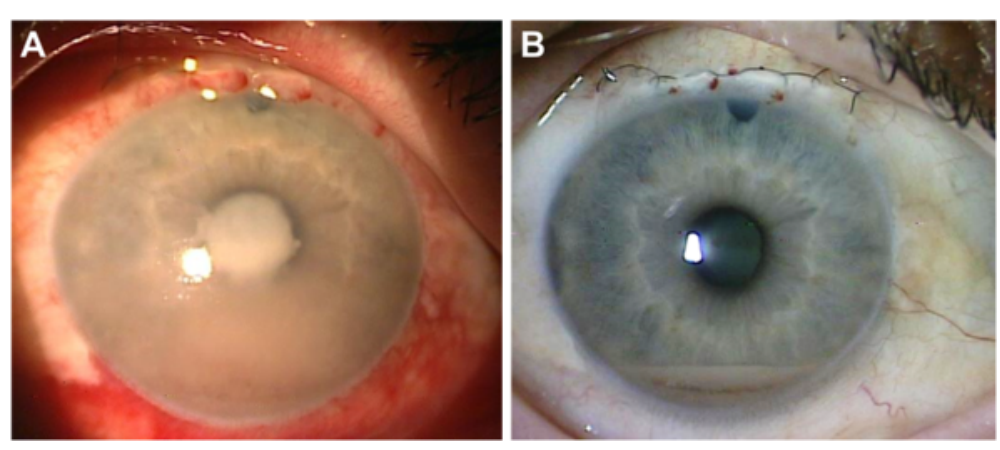

Fig. 1 a Slit-lamp photograph showing lymphoma involving the iris and anterior chamber (AC). There was diffuse conjunctival injection, a large hypopyon, fibrin over the pupil, and iris neovascularization (especially nasally). $\mathbf{b}$ Slit-lamp photograph taken 2 weeks after initiation of treatment shows a marked decrease in the conjunctival injection, AC inflammation, and hypopyon. The fibrin is no longer seen. Superior iridectomy and suture from prior trabeculectomy are seen 

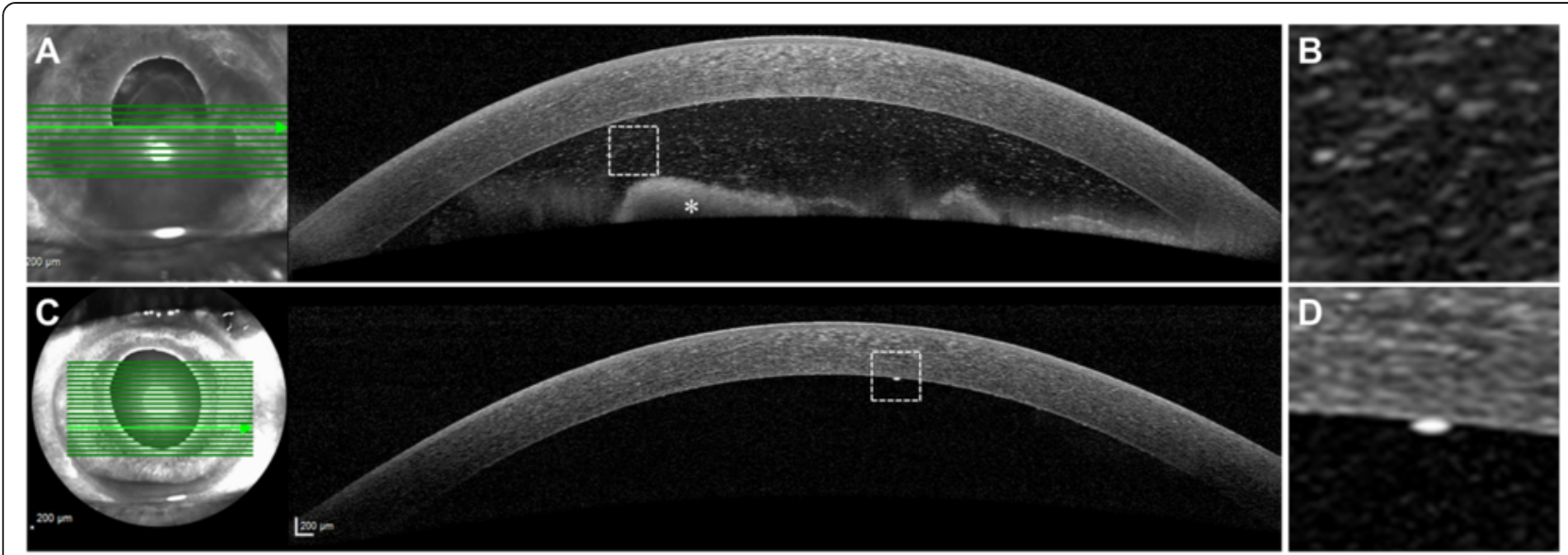

Fig. 2 a The anterior segment optical coherence tomography (AS-OCT) performed using the cornea protocol shows the presence of a large, irregular hyper-reflective cellular material floating in the anterior chamber (AC). $\mathbf{b}$ shows the magnified view of the lymphoma cells. c The follow-up AS-OCT scan shows a decrease in AC inflammation. $\mathbf{d}$ A single large granulomatous keratic precipitate is captured and its magnified view is shown

Ultrasound biomicroscopy (UBM) (Ellex Eye Cubed ${ }^{\text {tw }}$, Adelaide, Australia) findings were consistent with the AS-OCT, showing iris thickening in OD compared to OS (Fig. 4a, b).

At 1-week follow-up, BCVA improved to 20/50 in OD. The hypopyon and iris neovascularization markedly decreased. Evaluation of the posterior segment with fluorescein angiography did not demonstrate any abnormality. During the next 3 months, the patient received two additional injections of intravitreal rituximab at 6-week intervals and one additional injection of MTX and ranibizumab 6 weeks after presentation with interval improvement. AS-OCT demonstrated marked decrease in the AC cells (Fig. 2c). The iris thickening reduced following the combination chemotherapy (Fig. 4c-f). Systemic lymphoma demonstrated stabilization followed by improvement. Ibrutinib therapy was tapered to $420 \mathrm{mg} /$ day due to the concern of secondary myelodysplastic syndrome and an episode of neutropenic fever.
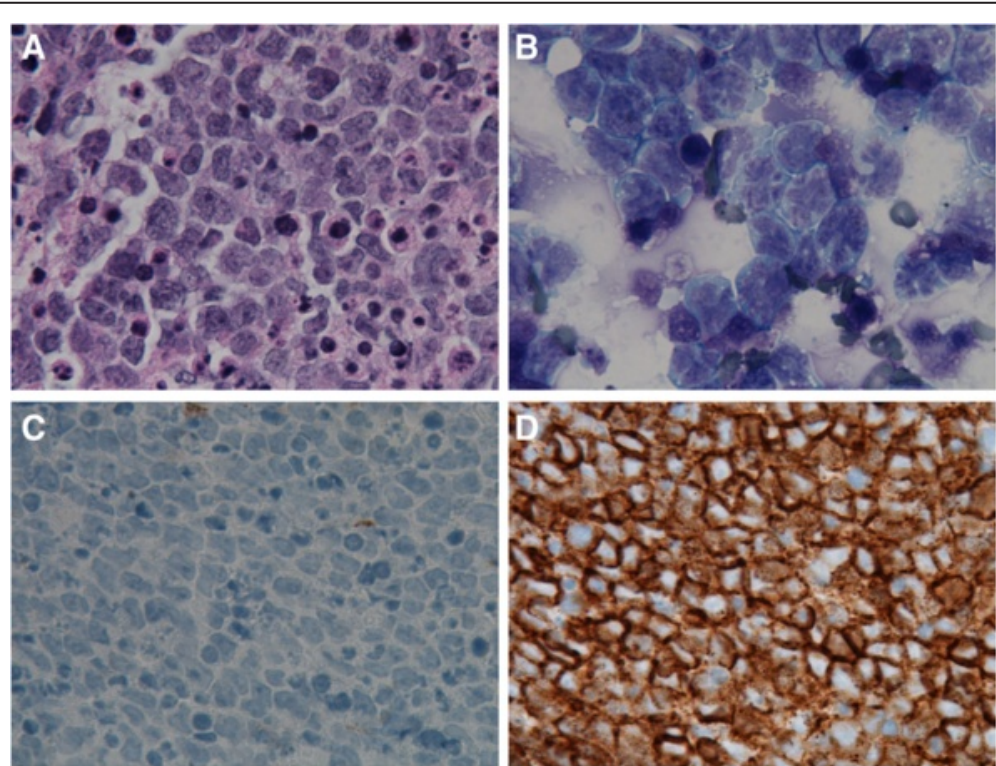

Fig. 3 a Photomicrograph of cell block showing cytological details of the anterior chamber fluid cells. a Hematoxylin and eosin (H\&E) stain (100x) demonstrates malignant lymphocytes. b Diff-Quik staining (100X) of the sample shows lymphoma cells with atypical, enlarged, irregular nuclei and increased N/C ratio. c Immunostaining with CD3 (100x) shows negative staining of the lymphoma cells. $\mathbf{d}$ Immunostaining with pan-B cell marker CD20 (100x) shows strong membranous staining. The findings on histopathology and the immunoprofile are consistent with the diagnosis of mantle cell lymphoma 

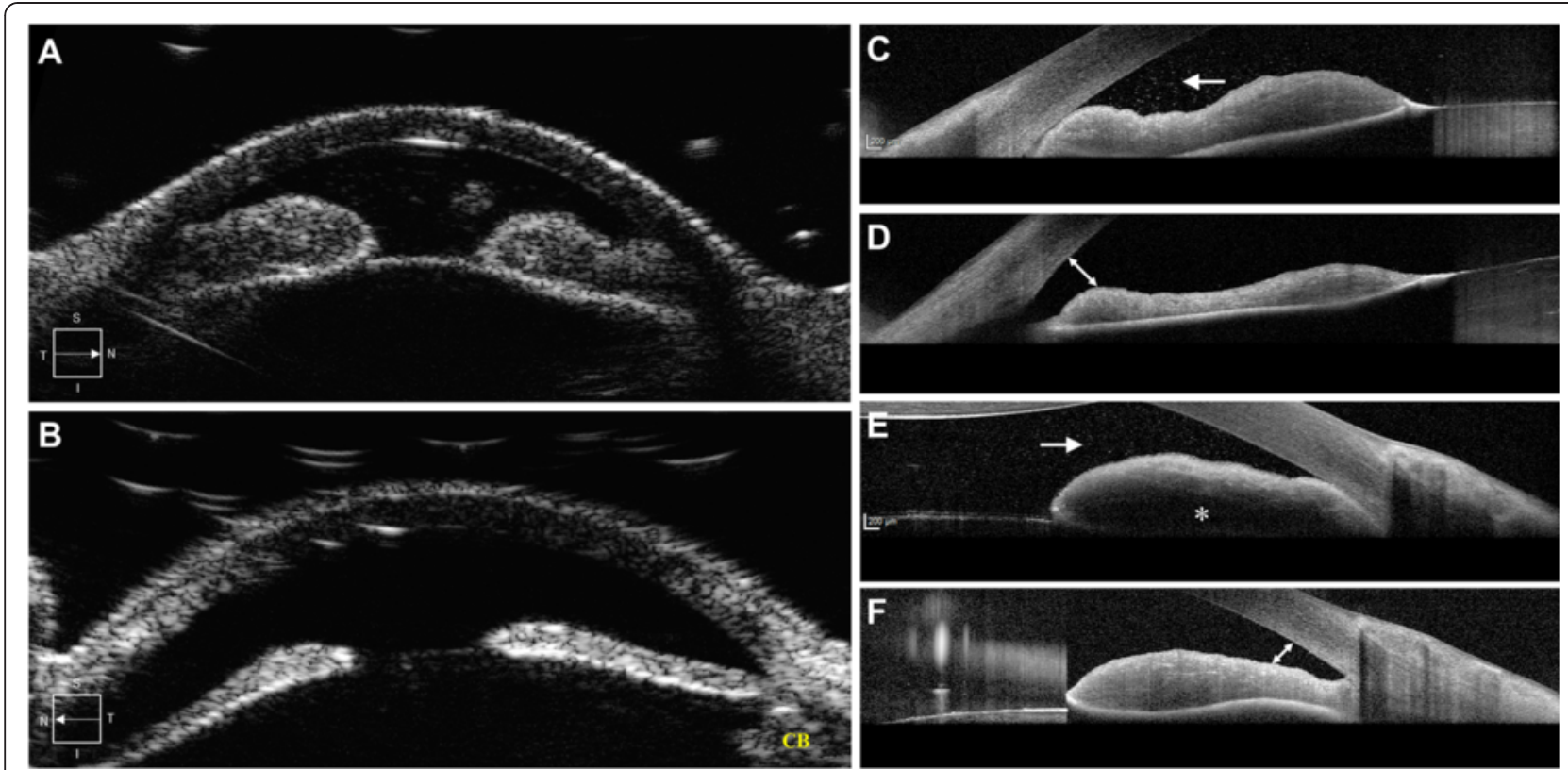

Fig. 4 a Ultrasound biomicroscopy (UBM) of the right eye shows secondary angle closure due to thickened iris. $\mathbf{b}$ UBM of the left eye shows normal appearance of the iris of the fellow eye with open angles. CB indicates the ciliary body. c AS-OCT of the right eye shows the pre-treatment state of the temporal iris. The white arrow indicates the floating lymphoma cells. The angle of the AC appears closed. $\mathbf{d}$ The appearance of the iris after institution of therapy reveals a decrease in the thickness and opening up of the angle (double-sided arrow). e The nasal iris shows partially visible posterior surface of the iris and internal hypo-reflectivity (asterisk) due to the increased tissue density anteriorly and poor penetration of the infrared rays through the thick iris. $\mathbf{f}$ The follow-up scan of the nasal iris after treatment shows reduction in the thickness and clearly visible posterior iris surface. The angle of AC appears open (double-sided arrow)

\section{Discussion}

To the best of our knowledge, uveal metastasis of MCL has been reported in only five cases in literature thus far $[6-8,16,17]$. Among these cases, isolated involvement of the anterior segment and iris was observed in two cases [6, 7]. In a report by Economou et al. [6], bilateral involvement of the iris was noted in a 71-year-old male with large, reddish tumor-like masses, anterior chamber inflammation, and hyphema. Reid et al. [7] reported unilateral granulomatous anterior chamber inflammation along with localized undulations of the iris and presence of aberrantappearing vasculature in an elderly male with systemic MCL. The diagnosis of intraocular MCL was confirmed using cytopathology in only one case by Economou et al. [6]. In all these case reports, the management of secondary iris lymphoma was limited to frequent topical steroids, systemic chemotherapy, or external beam radiation. Since the description of these cases, numerous systemic and local ocular chemotherapeutic strategies have evolved in the management of aggressive relapsing tumors with secondary ocular metastasis. The use of intravitreal chemotherapy along with systemic anti-cancer agents has not been described for the treatment of iris metastasis of MCL or other types of NHL.
Concurrent treatment with intravitreal MTX and rituximab has been successfully employed in the management of vitreoretinal NHL with few adverse events and remission achieved in more than two third of cases [10]. Similarly, for systemic therapy in NHL, rituximab is often combined with other chemotherapeutic agents given its anti-CD20 activity. In this case, we used intravitreal injections of both MTX and rituximab due to the extensive tumor load.

Ibrutinib has been approved by the United States Food and Drug Administration (US-FDA) for the treatment of MCL in patients who have received at least one prior therapy [12]. In our patient, chemotherapy with ibrutinib resulted in marked decrease in the systemic metastatic foci of MCL. However, the exact role of ibrutinib in reducing the ocular tumor load remains unclear.

UBM and AS-OCT complement each other in the diagnosis and management of non-pigmented iris tumors [18]. These techniques produce high-resolution images of the iris tumors resulting in excellent visualization of the tumor anatomy and measurement of tumor dimensions. Careful evaluation of morphological features of the iris on UBM and AS-OCT enabled effective monitoring of the treatment response in our patient (Figs. 2 and 4). To the best of our knowledge, the appearance of lymphoma cells on AS-OCT has not been 
characterized (Fig. 2). In this case, multimodal imaging allowed an objective assessment of reduction in the tumor load.

\section{Conclusions}

The index case describes successful initial management of anterior segment ocular metastasis in MCL with combined systemic and local ocular chemotherapeutic agents. The treatment regimen may be individualized; results on flow cytometry may guide the selection of appropriate pharmacologic agents after AC paracentesis. Iris metastasis can be well visualized using both UBM and AS-OCT, enabling serial objective assessments of the response to chemotherapy.

\section{Consent to publish}

The consent to publish has been obtained from the participant to report individual patient data.

\section{Abbreviations}

AC: anterior chamber; ASCT: autologous stem cell transplant; AS-OCT: anterior segment optical coherence tomography; BCVA: best-corrected visual acuity; BTK: Bruton's tyrosine kinase; CSF: cerebrospinal fluid; INV: iris neovascularization; IOP: intraocular pressure; MCL: mantle cell lymphoma; MRI: magnetic resonance imaging; MTX: methotrexate; NHL: non-Hodgkin's lymphoma; PET: positron emission tomography; UBM: ultrasound biomicroscopy.

\section{Competing interests}

The authors declare that they have no competing interests.

\section{Authors' contributions}

AA conceived the manuscript, performed the literature review, and participated in the clinical management of the case. MAS, WRR, LSJ, and $\mathrm{MH}$ participated in the clinical management of the case, acquisition of data, data analysis, and drafting the manuscript. PJB participated in the management of the case and drafting the manuscript. WWW participated in acquisition of data and drafting the manuscript. QDN provided the concept and design, was involved in the management of the case, provided intellectual content, and revised the manuscript. All authors read and approved the final manuscript.

\section{Acknowledgments}

None.

\section{Financial support/Proprietary interests}

All authors certify that they have no affiliations with or involvement in any organization or entity with any financial interest (such as honoraria; participation in speakers' bureaus; membership, employment, consultancies, stock ownership, or other equity interest; and expert testimony or patent-licensing arrangements) or non-financial interest (such as personal or professional relationships, affiliations, knowledge, or beliefs) in the subject matter or materials discussed in this manuscript.

This study is supported in part by an unrestricted grant from Research to Prevent Blindness (RPB) to the Truhlsen Eye Institute at the University of Nebraska Medical Center.

\section{Author details}

${ }^{1}$ Stanley M. Truhlsen Eye Institute, University of Nebraska Medical Center, 985540 Nebraska Medical Center, Omaha 68198-5540, NE, USA. Division of Hematology and Oncology, Department of Internal Medicine, University of Nebraska Medical Center, Omaha, NE, USA. ${ }^{3}$ Department of Pathology and Microbiology, University of Nebraska Medical Center, Omaha, NE, USA.

\section{References}

1. Cortelazzo S, Ponzoni M, Ferreri AJ, Dreyling M (2012) Mantle cell lymphoma. Crit Rev Oncol Hematol 82(1):78-101. doi:10.1016/ j.critrevonc.2011.05.001

2. Campo E, Rule S (2015) Mantle cell lymphoma: evolving management strategies. Blood 125(1):48-55. doi:10.1182/blood-2014-05-521898

3. Vose JM (2013) Mantle cell lymphoma: 2013 update on diagnosis, riskstratification, and clinical management. Am J Hematol 88(12):1082-1088. doi:10.1002/ajh.23615

4. Rasmussen P, Sjo LD, Prause JU, Ralfkiaer E, Heegaard S (2009) Mantle cell lymphoma in the orbital and adnexal region. Br J Ophthalmol 93(8):1047-1051. doi:10.1136/bjo.2008.146910

5. Mashayekhi A, Shields CL, Shields JA (2013) Iris involvement by lymphoma: a review of 13 cases. Clin Experiment Ophthalmol 41(1):19-26. doi:10.1111/ j.1442-9071.2012.02811.x

6. Economou MA, Kopp ED, All-Ericsson C, Seregard S (2007) Mantle cell lymphoma of the iris. Acta Ophthalmol Scand 85(3):341-343. doi:10.1111/ j.1600-0420.2006.00845.x

7. Reid JP, Puglis CL, Slagle WS (2014) Intraocular mantle cell lymphoma of the iris. Optom Vis Sci 91 (4 Suppl 1):S25-29. doi:10.1097/opx.0000000000000218

8. Chappelow AV, Singh AD, Perez VL, Lichtin A, Pohlman B, Macklis R (2008) Bilateral panocular involvement with mantle-cell lymphoma. J Clin Oncol 26(7):1167. doi:10.1200/jco.2007.14.2620

9. Franco P, Filippi AR, Ricca I, Raucci C, Ricardi U (2009) Eyelid localization in mantle cell lymphoma: long-lasting complete remission after surface brachytherapy. Tumori 95(3):385-388

10. Larkin KL, Saboo US, Comer GM, Forooghian F, Mackensen F, Merrill P, Sen HN, Singh A, Essex RW, Lake S, Lim LL, Vasconcelos-Santos DV, Foster CS, Wilson DJ, Smith JR (2014) Use of intravitreal rituximab for treatment of vitreoretinal lymphoma. Br J Ophthalmol 98(1):99-103. doi:10.1136/bjophthalmol-2013-304043

11. Frenkel S, Hendler K, Siegal T, Shalom E, Pe'er J (2008) Intravitreal methotrexate for treating vitreoretinal lymphoma: 10 years of experience. Br J Ophthalmol 92(3):383-388. doi:10.1136/bjo.2007.127928

12. Herrera AF, Jacobsen ED (2014) Ibrutinib for the treatment of mantle cell lymphoma. Clin Cancer Res 20(21):5365-5371. doi:10.1158/10780432.ccr-14-0010

13. Ritchie DS, Seymour JF, Grigg AP, Roberts AW, Hoyt R, Thompson S, Szer J, Prince HM (2007) The hyper-CVAD-rituximab chemotherapy programme followed by high-dose busulfan, melphalan and autologous stem cell transplantation produces excellent event-free survival in patients with previously untreated mantle cell lymphoma. Ann Hematol 86(2):101-105. doi:10.1007/s00277-006-0193-2

14. Martin N, Borchiellini D, Coso D, Gastaud L, Boscagli A, Saudes L, Re D, Gutnecht J, Garnier G, Petit E, Barriere J, Naman H, Rossignol B, Thyss A, Peyrade F (2015) High-dose chemotherapy with full BEAM followed by autologous stem cell transplantation is an effective treatment for elderly patients with poor-prognosis lymphoma. Leukemia \& lymphoma:1-26. doi:10.3109/10428194.2014.1001987

15. Rummel MJ, Niederle N, Maschmeyer G, Banat GA, von Grunhagen U, Losem C, Kofahl-Krause D, Heil G, Welslau M, Balser C, Kaiser U, Weidmann E, Durk H, Ballo H, Stauch M, Roller F, Barth J, Hoelzer D, Hinke A, Brugger W (2013) Bendamustine plus rituximab versus CHOP plus rituximab as first-line treatment for patients with indolent and mantle-cell lymphomas: an open-label, multicentre, randomised, phase 3 non-inferiority trial. Lancet 381(9873):1203-1210. doi:10.1016/s0140-6736(12)61763-2

16. Ahn ES, Singh AD, Smith SD (2010) Mantle cell lymphoma with uveal metastasis. Leuk Lymphoma 51(7):1354-1355. doi:10.3109/10428194.2010.486093

17. Rowley SA, Fahy GT, Brown LJ (2000) Mantle cell lymphoma presenting as a choroidal mass: part of the spectrum of uveal lymphoid infiltration. Eye (Lond) 14(Pt 2):241-244. doi:10.1038/eye.2000.63

18. Krema H, Santiago RA, Gonzalez JE, Pavlin CJ (2013) Spectral-domain optical coherence tomography versus ultrasound biomicroscopy for imaging of nonpigmented iris tumors. Am J Ophthalmol 156(4):806-812. doi:10.1016/ j.ajo.2013.05.025 